\title{
Adverse childhood experiences research: commonalities with similar, arguably identical, literatures and the need for integration
}

Andy P. Siddaway

\begin{abstract}
Summary
Adverse childhood experiences (ACES) and their impact cannot be clearly distinguished from other distressing life experiences. Much can be therefore be learned by integrating ACEs research with similar, well-established literatures. Future research needs to explain individual differences in relationships between ACES and particular variables and locate reliable and strong risk factors for ACEs themselves.
\end{abstract}

\section{Declaration of interest}

None.

\section{Keywords}

Post-traumatic stress disorder; bereavement; adverse childhood experiences (ACES); risk factor; trauma.

\section{Copyright and usage}

(C) The Royal College of Psychiatrists 2019
Scientific and clinical interest in adverse childhood experiences (ACEs) is burgeoning and has undoubtedly raised awareness of the potential impact of trauma and adversity and the importance of prevention and early intervention. The original ACEs study ${ }^{1}$ has been cited $\sim 8500$ times. Several subsequent studies suggest that enquiring about ACEs does not cause significant distress or increase service use, ${ }^{2}$ and that the observed relationships hold when controlling for demographic factors and social deprivation. ${ }^{3}$ If the influence of this growing area is to continue, several key issues now need to be addressed. This commentary outlines some ideas about how ACEs research and practice might be further evolved.

\section{Integration not separatism}

Research on ACEs is often being framed as an 'agenda' rather than a body of evidence. This is an understandable promotional and perhaps political tool but has separatist implications and is likely to attract criticism. That what happens to us - particularly in childhood - may have important consequences for our mental and physical health is not new to social workers, child mental health professionals, child development and trauma researchers, and many others. However, ACEs research appears to be garnering disproportionate policy attention and funding compared with related, similar fields (e.g. childhood trauma). Rather than creating a separate literature, ACEs research will be most likely to have an impact on the scientific knowledge base and be utilised to improve people's lives if it is integrated with the voluminous theoretical, empirical and treatment literatures on trauma, stress, meaning-making, complex/post-traumatic stress disorder (C/PTSD), coping and adjustment. Such an integration would make it more likely that a robust body of evidence is developed, avoid reinventing the wheel, and benefit all these literatures.

\section{Cautions for interpreting ACEs research}

One concern that might be offered at present is that the importance of ACEs in predicting physical and mental health outcomes is sometimes being exaggerated by some of the producers and consumers of this research. Very large studies ${ }^{4}$ and a meta-analysis of the ACEs literature ${ }^{5}$ indicate that the relationships between ACEs and physical and mental health variables tends to be relatively modest, even when four or more ACEs are considered. Odds ratios of $\sim 2$ have generally been observed, ${ }^{4,5}$ which indicates a twofold increase in particular physical and mental health variables, some of which are rare. A predictor that doubles or triples the risk of a rare outcome is not necessarily clinically important. There is an important difference between absolute risk and relative risk.

When interpreting ACEs research, stakeholders would also benefit from holding in mind the difference between statistical significance and effect sizes, and the different types of risk factor and the conclusions that can be drawn from each. A 'risk factor' is a type of correlate that precedes an outcome of interest in a longitudinal study; a 'causal risk factor' is a type of risk factor that is identified when the manipulation of a risk factor in an experimental design systematically changes an outcome of interest. ${ }^{6}$ Causal risk factors make important treatment targets; non-causal risk factors probably make less effective treatment targets; correlates may be ineffective treatment targets. Researchers, clinicians and policymakers must all be careful in how they interpret ACEs research and avoid drawing conclusions that go beyond the data.

\section{Exposure is not sufficient: a mantra to be appreciated}

Readers of ACEs research also need to keep in mind that the observed relationships are group-level (i.e. average) effects. The literature has yet to turn its attentions in any concerted way to explaining individual differences. A relevant mantra from the PTSD literature which conveys the complexity of relationships between particular experiences and the potential development of PTSD or other mental health problems is: 'exposure is not sufficient'. How, why, when, and for whom particular ACEs or other experiences can influence the onset and/or maintenance of particular problems is presumably changeable, complex, and multiply determined. Childhood PTSD research has generally demonstrated that an individual's interpretation of what happened to them, how they coped, and how their social environment responded, are far more important predictors than the objective severity of the trauma(s) experienced. ${ }^{7}$ The same may be true for ACEs.

Furthermore, it is difficult to clearly distinguish between life events, stressors, traumas, losses, and adversities, or between the impact of ACEs and adverse adult experiences (AAEs). For example, a recent meta-analysis demonstrated that PTSD 
symptoms were equally common following events defined as traumatic versus non-traumatic according to DSM-IV. ${ }^{8}$ This and other evidence meant that the DSM-5 Work Group considered removing the requirement of exposure to trauma from the PTSD diagnosis, so that the diagnosis focused entirely on trauma-related symptoms/impact. The point here is that many of the challenges that ACEs researchers face in conceptualising and researching ACEs have already been encountered and addressed by similar, well-established literatures, and there is therefore much to be gained by integrating ACEs research with those literatures and identifying common transdiagnostic underlying mechanisms.

\section{How robust is the ACEs literature?}

Another point for discussion is that the robustness of the ACEs literature is unclear, as some ACEs evidence is directly contradicted by evidence from other fields. For example, a meta-analysis of the ACEs literature found a very large odds ratio between ACEs and attempting suicide $(30.14),{ }^{5}$ whereas a meta-analysis of risk factors for suicide found an odds ratio of $\sim 1 .{ }^{9}$ Additionally, different authors have offered different lists of events that constitute ACEs, thus hindering comparisons and generalisations. None of these lists is comprehensive, and it does not seem feasible or beneficial to make them so, as there is potentially no limit to the stressors and challenges that may be faced in childhood and adulthood that directly or indirectly influence a person's mental and physical health. Bullying and parenting that is emotionally depriving, overprotective, critical or punitive are not considered ACEs and bereavement is often not considered an ACE. It is also important to note that, at present, different ACEs are weighted equally, such that an individual whose entire family was murdered and an individual whose father went to prison for shoplifting for food would both have experienced one ACE. Decades of trauma research ${ }^{9}$ indicates that not all events are equal and there is not a simple or 1:1 relationship between experiences and their impact. These issues clearly bring into question the use of ACE scores.

\section{Directions for future research}

These complex concerns all need to be borne in mind by stakeholders of ACEs research. As exposure to ACEs (and trauma) is near ubiquitous and unequally distributed, ${ }^{4,10}$ the ACEs literature now needs to focus on identifying mechanisms that explain individual differences in the associations between ACEs and mental and physical health variables (with implications for treatment), and locating reliable and strong risk factors for ACEs themselves (with implications for prevention and early intervention).
Lessons could be learned from the trauma and bereavement literatures in this regard, including how to differentiate prevention efforts and mental health services for youths and adults. Limited resources may be best dedicated to populations that are more likely to be exposed to multiple ACEs and traumas. Overall, it seems clear that much can be learned by incorporating the innovations and theoretical advances from similar, arguably identical fields into ACEs research. Doing so will maximise the research, policy and public health impact of this important field and will probably save money. To secure lifelong health, researchers, policymakers and mental health services should equally prioritise ACEs, child maltreatment, childhood trauma and social deprivation.

Correspondence: Andy P. Siddaway, (D) BSC (Hons), DClinPsy, PhD, Clinical Psychologist, Behavioural Science Centre, Stirling Management School, 3B55 cottrell Building, University of Stirling, Stirling FK9 4LA, UK. Email: andy.siddaway@stir.ac.uk First received 17 Apr 2019, final revision 25 Jun 2019, accepted 3 Jul 2019

\section{References}

1 Felitti VJ, Anda RF, Nordenberg D, Williamson DF, Spitz AM, Edwards V, et al. Relationship of childhood abuse and household dysfunction to many of the leading causes of death in adults: the Adverse Childhood Experiences (ACE) Study. Am J Prev Med 1998; 14: 245-58.

2 Edwards VJ, Dube SR, Felitti VJ, Anda RF. It's OK to ask about past abuse. Am Psychol 2007; 62: 327-8.

3 Bellis M, Hughes K, Leckenby N, Perkins C, Lowey H. National household survey of adverse childhood experiences and their relationship with resilience to health-harming behaviors in England. BMC Med 2014; 12: 72.

4 Kessler RC, McLaughlin KA, Green JG, Gruber MJ, Sampson NA, Zaslavsky AM, et al. Childhood adversities and adult psychopathology in the WHO World Mental Health Surveys. Br J Psychiatry 2010; 197: 378-85.

5 Hughes K, Bellis MA, Hardcastle KA, Sethi D, Butchart A, Mikton C, et al. The effect of multiple adverse childhood experiences on health: a systematic review and meta-analysis. Lancet Public Health 2017; 2: e356-66.

6 Kraemer HC, Kazdin AE, Offord DR, Kessler RC, Jensen PS, Kupfer DJ. Coming to terms with the terms of risk. Arch Gen Psychiatry 1997; 54: 337-43.

7 Trickey D, Siddaway AP, Meiser-Steadman R, Serpell L, Field AP. A metaanalysis of risk factors for posttraumatic stress disorder in children and adolescents. Clin Psychol Rev 2012; 32: 122-38.

8 Larsen SE, Pacella ML. Comparing the effect of DSM-congruent traumas vs. DSM-incongruent stressors on PTSD symptoms: a meta-analytic review. J Affect Disord 2016; 38: 37-46.

9 Franklin JC, Ribeiro JD, Fox KR, Bentley KH, Kleiman EM, Huang X, et al. Risk factors for suicidal thoughts and behaviors: a meta-analysis of 50 years of research. Psychol Bull 2017; 143: 187-232.

10 Benjet C, Bromet E, Karam EG, Kessler RC, McLaughlin KA, Ruscio AM, et al. The epidemiology of traumatic event exposure worldwide: results from the World Mental Health Survey Consortium. Psychol Med 2016; 46: 327-43 